\title{
LYMPHOEPITHELIOMA LIKE CARCINOMA OF KIDNEY - A RARE ENTITY.
}

Susruthan $\mathrm{M}^{1}$, Rajesh $\mathrm{H}^{2}$, Rajendiran $\mathrm{S}^{3}$

\section{HOW TO CITE THIS ARTICLE:}

Susruthan M, Rajesh H, Rajendiran S. "Lymphoepithelioma like carcinoma of kidney - a rare entity". Journal of Evolution of Medical and Dental Sciences 2013; Vol. 2, Issue 47, November 25; Page: 9163-9166.

INTRODUCTION: Lymphoepithelioma, otherwise known as poorly differentiated carcinoma commonly occurs in nasopharynx. Tumors with similar morphology occur at salivary gland, stomach, thymus, urogenital system and lung which are known as Lymphoepithelioma like carcinoma (LELC) Its incidence in kidney is extremely rare. We describe here a report, to our knowledge, the seventh case of Lymphoepithelioma like carcinoma occurring in kidney (LELC).

CASE PRESENTATION: A 65 years old woman presented with left flank pain for three months. CT abdomen revealed a mass of size $5.2 \times 5 \times 4 \mathrm{~cm}$ in the upper pole of kidney with heterogeneous contrast enhancement. Left radical nephrectomy was performed. On microscopy two cell populations were present, one being large atypical cells positive for cytokeratin and other being lymphoid cells showing CD 3 positivity. A final diagnosis of lymphoepithelioma like carcinoma of kidney pT3 N2a cM0 was rendered.

DISCUSSION: LELC is distinguished from xanthogranulomatous pyelonephritis by the nests of cytokeratin positive large cells with prominent nucleoli in a lymphoid background. Cytokeratin negativity in Non Hodgkin's Lymphoma helps in the distinction of LELC from lymphomas. LELCs are distinguished from collecting duct carcinoma by the absence of desmoplasia and marked cytological atypia. The right diagnosis is vital since treatment regimens, prognostic factors and survival rates vary.

\section{CASE REPORT:}

INTRODUCTION: Lymphoepithelioma, otherwise known as poorly differentiated carcinoma is a common tumor occurring in the nasopharynx, related in most cases to Epstein Barr virus. Tumors with similar morphology in other sites such as salivary gland, stomach, thymus and lung are known as Lymphoepithelioma like carcinoma (LELC) ${ }^{1}$. LELC rarely may present in the urogenital system, the common site among them being the urinary bladder. Its incidence in kidney is extremely rare ${ }^{2}$ and we report, to our knowledge, the seventh case of Lymphoepithelioma like carcinoma occurring in kidney (LELC).

CASE HISTORY: A 65 year old diabetic woman presented with left flank pain for three months. She had attained menopause ten years back. Hematological parameters were within normal limits. Echocardiography showed mild concentric left ventricular hypertrophy with aortic sclerosis. Computerised tomography (CT) of abdomen revealed a fairly circumscribed mass in the upper pole of kidney measuring $5.2 \times 5 \times 4 \mathrm{~cm}$ with medial and superior extension. On contrast CT scan the tumor was heterogeneously enhancing. Left radical nephrectomy was performed.

On gross examination, the left radical nephrectomy specimen measured $14 \times 8 \times 5 \mathrm{~cm}$ and weighed 410 grams. External surface showed a nodular mass involving the upper pole. Cut surface 
showed fairly circumscribed grey white, soft to rubbery tumor measuring $5 \times 5 \times 4 \mathrm{~cm}$ with yellow specks and tiny cysts. Tumor was seen extending into the renal pelvis and the capsule [Fig 1]. The adjacent kidney parenchyma was slightly compressed by the tumor and otherwise was unremarkable. Adrenal glands, renal vessels and the ureter were free of tumor.

Microscopically the tumor showed two population of cells [Fig. 2].One of them being, large atypical cells showing hyperchromatic nuclei with prominent nucleoli and moderate amount of eosinophilic cytoplasm arranged predominantly in a syncytial pattern. Mild to moderate pleomorphism with frequent mitotic figures of 5per 10 high power fields were observed. The second population was made up of predominantly lymphocytes, few plasma cells and scattered histiocytes. Many lymphocytes were also seen infiltrating into the islands of large cells. There was no evidence of significant necrosis or hemorrhage. The tumor was seen extending into the renal pelvis (pT3a). Four out of the eight lymph nodes dissected were positive for tumour (pN2).

Immunohistochemically, cytokeratin was positive in the atypical large cell component [Fig. 3]. Most of the lymphoid cells were positive for CD 3[Fig 4] and a few of them were CD20 positive.

After reviewing Hematoxylin and Eosin (H\&E) and Immunohistochemical stains, a final diagnosis of lymphoepithelioma like carcinoma of kidney (pT3 N2a cM0) was rendered. All the surgical margins were free from tumor.

DISCUSSION: Tumors showing the histological features of lymphoepithelioma outside the nasopharynx are rare. LELC has been reported in the literature involving the renal parenchyma, pelvis, ureter and bladder, the latter being the most common site in the urinary system. Lymphoepithelioma like carcinoma has to be distinguished on one hand from non tumorous conditions such as xanthogranulomatous pyelonephritis and on the other hand from high grade tumors like Non Hodgkin's lymphoma (NHL) and Collecting duct carcinoma1,3.

The prominent lymphoid background in the tumour presents a challenge for the pathologists in distinguishing LELC from inflammatory lesions such as xanthogranulomatous pyelonephritis. This distinction is essential owing to the obvious difference in prognosis and management of the above two conditions. The presence of nests of cytokeratin positive large cells with prominent nucleoli in a lymphoid back ground will fetch the correct diagnosis of LELC.

Immunohistochemistry helps in the distinction of LELC from lymphomas, since the treatment regimens, prognostic factors and survival rates vary among these tumours. . The large atypical cells of LELC are negative for CD 45 (Leucocyte Common Antigen), whereas they are positive for cytokeratin. The lymphoid population in LELC is of polyclonal in type. All of them are CD 45 positive and most of them are CD 3 positive with scattered CD 20 positivity, indicating mixture of $\mathrm{T}$ cells with a few $\mathrm{B}$ cells. In contrast, the lymphoma cells are monoclonal, all of them are positive for CD 45 and majority of them are CD 20 positive as most of the reported NHL of kidney are of B cell type. The lymphoid cells are negative for cytokeratin.2,3

LELCs can be distinguished from other high grade carcinomas such as collecting duct carcinoma by the absence of desmoplasia and marked cytological atypia. The gross appearance of the tumor also can give some clues as the LELC will be firm to rubbery in consistency (akin to lymphoma or tumors with lymphoid infiltrate) with less of necrosis and haemorrhage. Classic and high grade renal cell carcinoma will have variegated look with areas of haemorrhage and necrosis. 
The exact etiology and pathogenesis of LELC involving the kidney and the urinary system is unknown. In the kidney they should be most probably arising from the pelvi-calyceal urothelium rather than from the renal epithelium. In most of the reported cases, they usually present as a higher stage lesion. Despite high stage and poor differentiation, the prognosis after surgery with and with out adjuvant therapy has been extraordinarily favorable at least in the documented bladder LELC 4. The exact prognosis of renal LELC is unknown.

Our patient is on adjuvant chemotherapy and is doing well at the sixth month follow up visit.

\section{REFERENCES:}

1. R. J. Cohen, J. C. Stanley, H. J. S. Dawkins. Lymphoepithelioma like carcinoma of the renal Pelvis Pathology 1999: 31(4); p434-35.

2. Delia Perez-Montiel, Paul E Wakely Jr, Ondrej Hes, Michal Michal and Saul Suster. High-grade urothelial carcinoma of the renal pelvis- a clinicopathologic study of 108 cases with emphasis on unusual morphologic variants. Mod Pathol 2006:19;p 494-503.

3. Haga. K, Aoyagi T, Kashiwagi A, Yamashiro K, Nagamori S. Lymphoepithelioma-like Carcinoma of the Renal Pelvis. Korean J Urol 2008: 49; p 461-463.

4. Tamas EF, Nielsen ME, Schoenberg MP, Epstein JI. Lymphoepithelioma like carcinoma of the urinary tract -a clinicopathological study of 30 pure and mixed cases. Mod Pathol 2007: 20; $p$ 828-34.
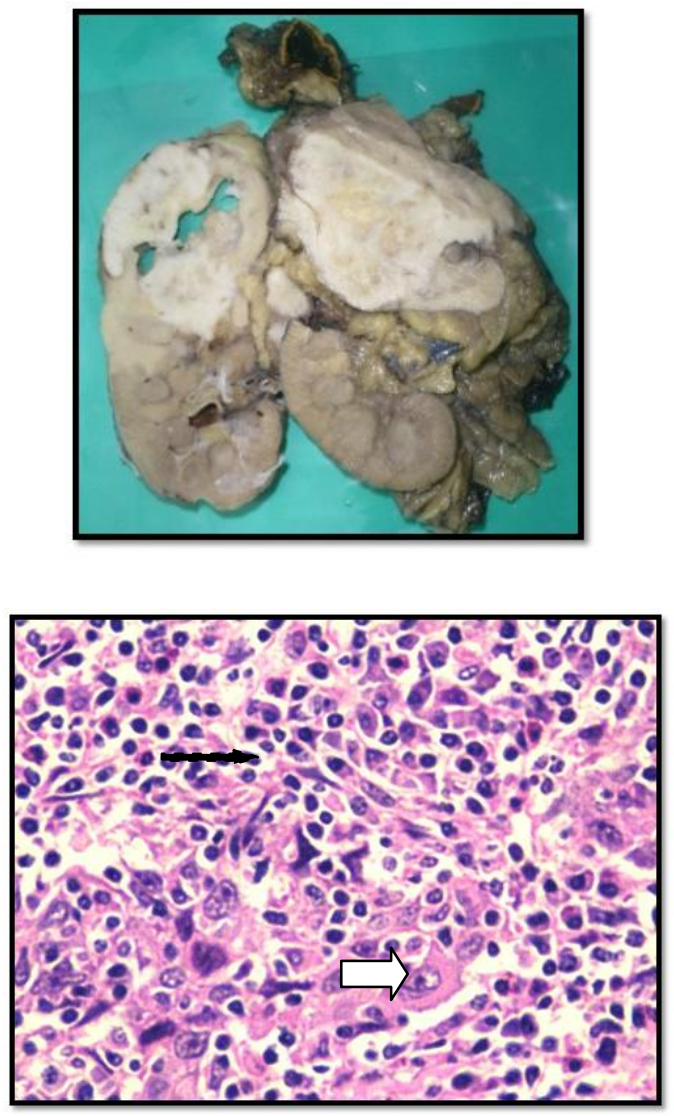

Fig. 1: Cut surface of nephrectomy specimen showing more or less circumscribed grey white tumor in the upper pole of the kidney occupying cortex and pelvis.
Fig. 2: Large atypical cells showing prominent nucleoli (white arrow) and arranged in syncytial pattern with interspersed lymphocytes(black arrow) [x100, H \& E]. 


\section{CASE REPORT}

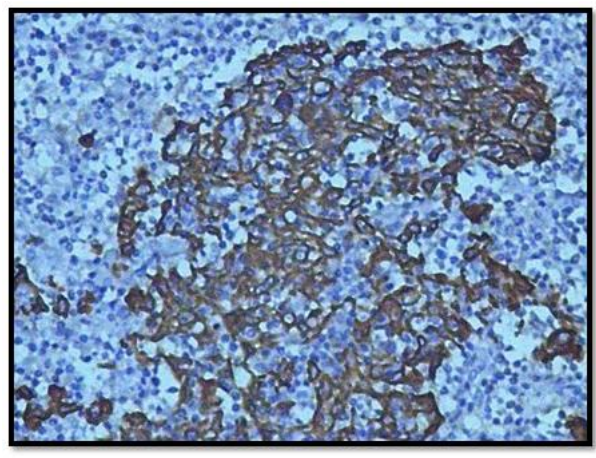

Fig. 3: Immunohistochemistry for cytokeratin showing cytoplasmic positivity in the large atypical cells and negative staining of lymphocytes in the background [x 100]

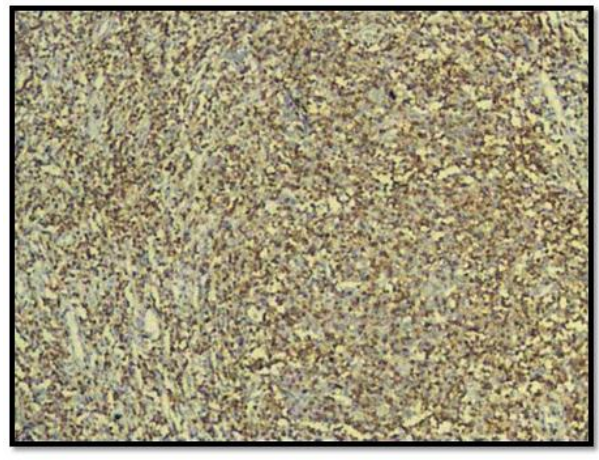

Fig. 4: Immunohistochemistry for CD 3 showing diffuse membrane positivity of the lymphoid cells [ $x 100]$.

\section{AUTHORS:}

1. Susruthan M.

2. Rajesh $\mathrm{H}$.

3. Rajendiran S.

\section{PARTICULARS OF CONTRIBUTORS:}

1. Assistant Professor (Selection Grade), Department of Pathology, Sri Ramachandra Medical College \& Research Institute.

2. Resident, Department of Pathology, Sri Ramachandra Medical College \& Research Institute.

3. Professor, Department of Pathology, Sri Ramachandra Medical College \& Research Institute.

\section{NAME ADDRESS EMAIL ID OF THE CORRESPONDING AUTHOR:}

Dr. M. Susruthan,

G2, Sri Varsha, 34 \& 35,

Krishna Street, Rajeswari Avenue, Madhananthapuram, Chennai - 600125.

Email -susruthansak@gmail.com

Date of Submission: 07/11/2013.

Date of Peer Review: 08/11/2013.

Date of Acceptance: 15/11/2013.

Date of Publishing: 20/11/2013 